Association for Information Systems AIS Electronic Library (AISeL)

Wirtschaftsinformatik Proceedings 2001

Wirtschaftsinformatik

September 2001

\title{
CSCL-interdisziplinär: Synergie oder Reibungsverluste?
}

Udo Hinze

Fachhochschule Stralsund, udo.hinze@fh-stralsund.de

Gerold Blakowski

Fachhochschule Stralsund, gerold.blakowski@fh-stralsund.de

Birgit Gaiser

Fachhochschule Brandenburg, gaiser@fh-brandenburg.de

Werner Beuschel

Fachhochschule Brandenburg, beuschel@fh-brandenburg.de

Follow this and additional works at: http://aisel.aisnet.org/wi2001

\section{Recommended Citation}

Hinze, Udo; Blakowski, Gerold; Gaiser, Birgit; and Beuschel, Werner, "CSCL-interdisziplinär: Synergie oder Reibungsverluste?" (2001). Wirtschaftsinformatik Proceedings 2001.27.

http://aisel.aisnet.org/wi2001/27

This material is brought to you by the Wirtschaftsinformatik at AIS Electronic Library (AISeL). It has been accepted for inclusion in Wirtschaftsinformatik Proceedings 2001 by an authorized administrator of AIS Electronic Library (AISeL). For more information, please contact elibrary@aisnet.org. 
In: Buhl, Hans Ulrich, u.a. (Hg.) 2001. Information Age Economy; 5. Internationale Tagung Wirtschaftsinformatik 2001. Heidelberg: Physica-Verlag

ISBN: 3-7908-1427-X

(C) Physica-Verlag Heidelberg 2001 


\title{
CSCL-interdisziplinär: Synergie oder Reibungsverluste?
}

\author{
Udo Hinze, Gerold Blakowski
}

Fachhochschule Stralsund

\author{
Birgit Gaiser, Werner Beuschel
}

Fachhochschule Brandenburg

Zusammenfassung: Eine interdisziplinäre Ausrichtung von Projekten, die sich mit CSCL (Computer Supported Collaborative Learning) beschäftigen, wurde bisher nur ansatzweise realisiert. Dies überrascht um so mehr, als die Vorteile für das kollaborative, computergestützte Lernen evident erscheinen. Aus diesem Grund wurde im Rahmen des Bundesleitprojektes „Virtuelle Fachhochschule" zwischen den Fachhochschulen in Brandenburg und Stralsund ein CSCL-Projekt mit interdisziplinärem Ansatz konzipiert und durchgeführt. Eingebettet in ein stringentes didaktisches Grundkonzept wurde eine weitgehend offene und interdependente Aufgabe gestellt, deren Lösung von den Studierenden an beiden Standorten gemeinsam erarbeitet werden musste.

Die Untersuchung bezog sich auf mehrere Faktoren zur Gestaltung von CSCLProjekten. Im Mittelpunkt stand die Auswirkung der Heterogenität bzw. Interdisziplinarität. Ein weiterer Schwerpunkt war die Erforschung der Konflikte, aber auch der explanativen Prozesse, die sich in den Gruppen vollzogen. Weiterhin wurde der Umgang der Studierenden mit Autonomie und Interdependenz der Aufgabenstellung untersucht. Von Interesse waren dabei Fragen wie:

- Existieren Stereotype und wie manifestieren sie sich in der Zusammenarbeit?

- Gibt es einen hohen Anteil an leistungsfördernden explanativen Prozessen?

- Überwiegen in der interdisziplinären Zusammenarbeit die Gewinne durch Synergie oder die Reibungsverluste?

- Wie organisieren sich die Gruppen bei einer weitgehend offen und interdependent gestalteten Aufgabenstellung?

Zusammengefasst trafen grundlegende Annahmen bezüglich etwa der Stereotype $z u$. Diese waren in hohem Maße latent vorhanden, wirkten sich aber nur indirekt auf die Zusammenarbeit aus. Vielmehr zeigte sich Dominanz, die vor allem durch höhere technische und inhaltliche Kompetenz, aber auch durch organisatorische Aspekte zu erklären ist. Die Vorteile, die sich prinzipiell in interdisziplinären Gruppen zeigen können (z.B. Explanation, Elaboration), fanden sich nicht in dem 
erwarteten Maße wieder. Explanative Prozesse waren beispielsweise eher die Ausnahme. Die Autonomie sowohl bezüglich der Aufgabe als auch im Hinblick auf die Kommunikationsmöglichkeiten wurde insgesamt von den Studierenden umfangreich genutzt. Überforderung durch zuviel Autonomie und daraus resultierende Demotivation wurde nur zu Beginn des Kooperationsprojektes beobachtet.

Schlüsselworte: CSCL, Konstruktivismus, kooperatives Lernen, Lernaufgabengestaltung, Fallstudie kooperatives Lernen, Telelearning

\section{Theoretischer Hintergrund - Möglichkeiten und Grenzen der Erfassung von Einflußfaktoren}

CSCL (Computer Supported Collaborative Learning) ist wissenschaftlich ein weitgehend unterbestimmtes Feld. Dieser Umstand liegt nicht primär darin begründet, dass das Forschungsfeld und interdisziplinäre wissenschaftliche Beschäftigung mit CSCL noch relativ jung ist. Ursache ist vielmehr die Vielzahl an interdependenten Variablen, die bei Konzeption und Durchführung von kooperativen Lernprozessen zu beachten sind. Diese Variablenvielfalt macht es relativ schwierig, generelle kausale Zusammenhänge zwischen der Gestaltung der Variablen und den Ergebnissen wie etwa dem Lernerfolg zu finden [Dill95].

Um die einzelnen Faktoren und deren Wirkung genauer auszuarbeiten und wissenschaftlich zu einem stringenteren Konzept zu kommen, schlägt z.B. Salomon [Salo95] vor, nur einzelne Faktoren zu variieren und deren Wirkung isoliert zu erforschen. Nach diesem Kausalmodell sollten dann die Schlussfolgerungen zu einem Erkenntnisfortschritt beitragen. In der Praxis sind diese Versuche relativ selten. Es existiert vielmehr eine Vielzahl von Projekten mit unterschiedlichem Fokus. Insgesamt sind die Projekte und die Forschungsergebnisse nur bedingt vergleichbar. Dies gilt schon im Hinblick auf die Forschungsperspektive. So betrachten die dem soziokognitiven Ansatz nach Piaget [Piag26] folgenden Wissenschaftler primär die Resultate der Kooperation. Im Gegensatz dazu untersuchen die Vertreter der soziokulturellen Perspektive nach Vygotsky [Vygo62] vor allem die (Inter)aktionsprozesse während der Gruppenarbeit.

Eine reine Faktorenanalyse bleibt deshalb meist illusorisch. Aus dieser Einsicht resultiert die Tendenz hin zu einer prozessorientierten Sicht auf die Rolle der Variablen im Interaktionsprozess [Dill95] sowie zu „lokalen” Theorien [MaRe92].

Insgesamt kann der Anspruch auf eindimensionale Erklärungsmuster nicht eingelöst werden. Die Feststellung ,it may be time to stop looking for general effects of collaboration" [Dill95] ist somit zutreffend. Dies indiziert, dass für die vorliegende Untersuchung ein qualitativer Ansatz angemessen ist. Der teilweise unbe- 
friedigende Stand der Forschung bedeutet aber nicht, dass quasi im theoriefreien Raum operiert werden muss, wie im folgenden erklärt wird.

\section{Grundannahmen und Fragestellungen}

Die Grundannahmen, die die Basis für das didaktische Konzept bildeten, resultierten zum einen aus den Ergebnissen der CSCL-Forschung, zum anderen aus konstruktivistischen Implikationen, die im folgenden skizziert werden. Grundlegende konstruktivistische Annahme ist, dass Wissen von den Lernern aktiv und in der Regel im sozialen Kontext konstruiert wird. Auf passive Rezeption der Lerner aufbauende Konzepte werden zunehmend hinterfragt. Grundlage der Wissenskonstruktion sind problemorientierte, authentische Aufgaben, die in ihrer Komplexität an das Vorwissen der Lerner anschließen. Um die selbstgesteuerte Wissenskonstruktion zu fördern, ist zudem ein adäquates Maß an Selbstorganisation und steuerung des Lernens zu ermöglichen. Diese Autonomie gilt sowohl im Hinblick auf die Aufgabenstellung, als auch für die technischen Möglichkeiten zur Kommunikation.

Neben dem didaktischen Prinzip der Autonomie wurde insbesondere die Heterogenität der interdisziplinären Gruppen und die daraus resultierenden Möglichkeiten zur Explanation berücksichtigt und daraus jeweils Fragestellungen entwickelt, die im Rahmen der Fallstudie untersucht wurden.

\subsection{Heterogenität}

Die Wirkung der Heterogenität der Teilnehmer in Gruppen gilt als einer der am dezidiertesten untersuchten Einflussfaktoren [Dill95]. Die Heterogenität wurde bereits auf vielen Feldern - z.B. Geschlecht, Status, Introversion vs. Extroversion, Motivation, Status etc.- untersucht [Webb91].

Heterogenität in Gruppen ist nicht per se ein Garant für hohe bzw. höhere Leistungen. Vielmehr muss ein spezifisches Maß an Differenzen bzw. eine optimale Heterogenität [DiSc95] vorhanden sein. Doch auch bei optimaler Heterogenität ist eine eindimensionale Kausalzuschreibung unmöglich. Heterogene Gruppen sind etwa für bestimmte Aufgaben (z.B. Problemlösungsaufgaben) besser geeignet als homogene, für andere hingegen schlechter.

Eine mögliche Erklärung, warum heterogene Gruppen unter geeigneten Umständen effektiver als homogene sind, bezieht sich auf den Wissensbestand in heterogenen Gruppen. Das Wissen der einzelnen Teilnehmer in heterogenen Gruppen (Abb. 2) ist im Gegensatz zu homogenen Gruppen von unterschiedlicher Qualität und Quantität (Abb. 1). Vereinfacht betrachtet sieht der Vergleich der Wissensverteilung etwa so aus: 


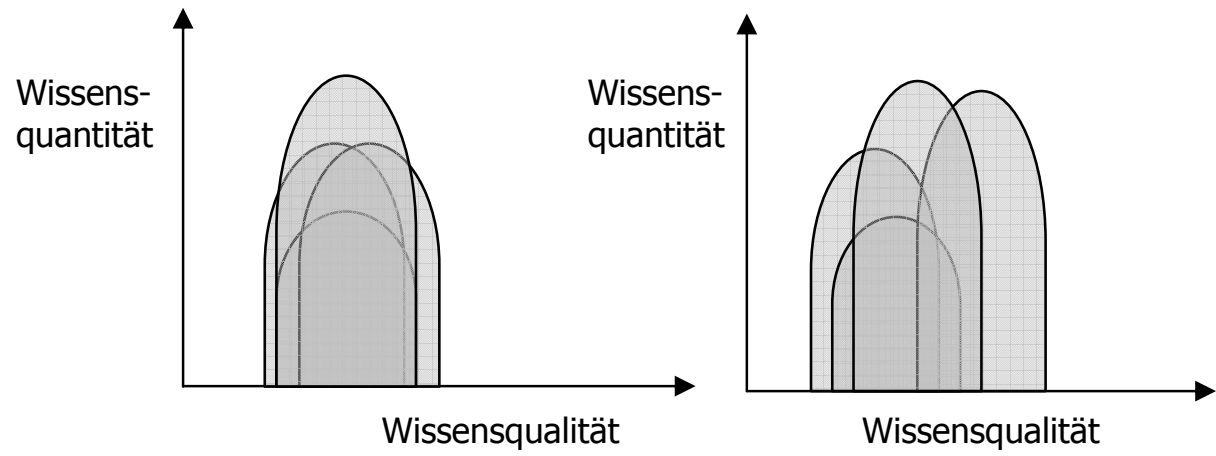

Abbildung 1: leistungshomogene Gruppen

Abbildung 2: leistungsheterogene Gruppen

Interdisziplinäre Gruppen stellen einen Sonderfall leistungsheterogener Gruppen dar. In leistungsheterogenen Gruppen haben die Teilnehmer ein divergentes Wissen bezüglich der Aufgabe. Grundsätzlich handelt es sich aber jeweils um den gleichen Wissensbestand, der bei den Teilnehmer in unterschiedlichen Ausmaß vorhanden ist. Dies liegt vor allem an den individuellen Kompetenzen, was sich auch auf den Status, d.h. z.B. auf Anerkennung und Akzeptanz des Einzelnen in der Gruppe auswirkt. In interdisziplinären Gruppen ist hingegen das Wissen in den jeweiligen Fachgebieten oft unterschiedlich verteilt (Abb. 3).

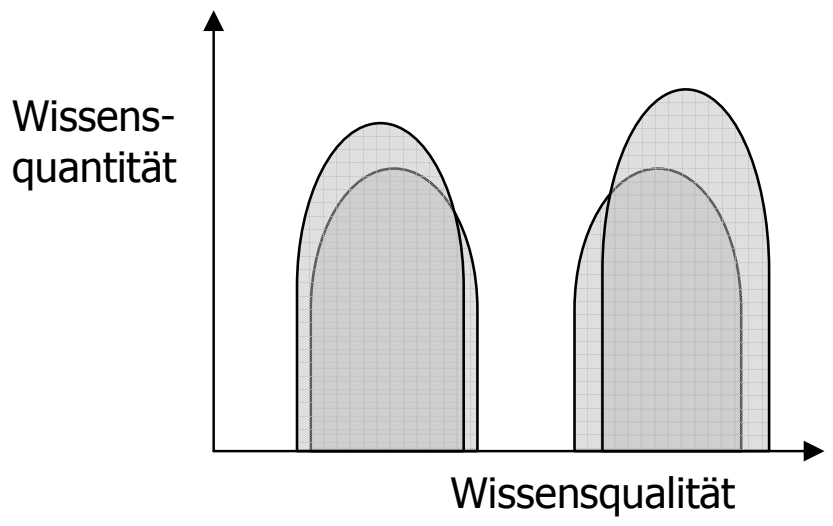

Abbildung 3: Wissensverteilung in interdisziplinären Gruppen

Ist also in den traditionellen leistungsheterogenen Gruppen das Kompetenzniveau auf einem Gebiet unterschiedlich hoch, so ist es bei interdisziplinären Gruppen auf mehreren Gebieten divergent. Diese multiple Divergenz bewirkt, dass die Teilnehmer jeweils sowohl die Rolle des Lehrers als auch die des Rezipienten ein- 
nehmen können. Prinzipiell sollte dieses statusunabhängige und gleichberechtigte Miteinander damit noch effektiver sein. Allerdings bleibt zu beachten, ob die Differenzen nicht als zu groß empfunden werden, so dass die Interaktion eher behindert wird.

Inwieweit sich die Differenzen tatsächlich als statusunabhängig erwiesen und ob die Differenzen optimal oder eher zu groß waren, war eine der Hauptfragen im Rahmen der Studie.

\subsection{Explanation, Elaboration und Konflikte}

Trotz der Evidenz der Überlegungen zur prinzipiellen Effizienz insbesondere interdisziplinärer Gruppen, muss beachtet werden, dass das divergente Wissen in den Gruppen durch Interaktion auch kommuniziert werden muss. Diese Interaktion ist eine der zentralen Begründungen für kooperatives Lernen aus kognitiver Sicht. Grundsätzlich wird davon ausgegangen, dass neue Informationen erst durch kognitive Umstrukturierungs- oder Elaborationsprozesse mit den vorhandenen Schemata verknüpft werden können. Neue Information wird begriffen, indem sie viabel in die kognitiven Schemata eingefügt wird.

Einer der effektivsten Elaborationsprozesse, mit denen der Lernstoff begriffen und an vorhandene Informationen angeknüpft werden kann, ist die Präsentation des Lernstoffs aus eigener Sicht; Lehren ist damit die effektivste Form des Lernens. Konzeptualisiert wird diese Tatsache als Perspektive der kognitiven Elaboration [Slav93]. Die Elaborationsprozesse sind allgemein in kooperativen und speziell in leistungsheterogenen Szenarien eine der Determinanten des Lernerfolgs.

Nach der Metaanalyse von Webb [Webb91] hat aber die Explanation auf den Lernerfolg des Rezipienten nur geringe bzw. keine Auswirkungen. Die Vorteile liegen vielmehr eindeutig bei der Person, die die Erläuterungen gibt. Da die Rollenverteilung bei leistungsheterogenen Gruppen eindeutig und eindimensional ist, profitieren die ohnehin Kompetenten von der Gruppenarbeit. In interdisziplinären Gruppen kehrt sich dieses Verhältnis hingegen ständig um und die Rollenverteilung wechselt permanent. Prinzipiell sollte dies für alle Beteiligten einen höheren Lerneffekt bringen. Allerdings dürfen die Differenzen nicht zu groß sein und es muss zudem die individuelle Bereitschaft zur Explanation bestehen. Wenn außerdem kein intrinsischer bzw. extrinsischer Anreiz zur Interaktion besteht, bleibt das Konzept der Explanation Makulatur.

Wenn zudem im Sinne der Perspektive von Piaget auf die Fruchtbarkeit von Sachkonflikten in Gruppen hingewiesen wird, ist darauf zu achten, wie sich diese Konflikte in interdisziplinären Gruppen manifestieren. Sozialpsychologische Aspekte wie Vorurteile und Stereotypen können hier eine wesentliche Rolle spielen. Damit können Abneigungen und Konflikte leicht auf persönlicher Ebene entstehen bzw. 
auf zwischenmenschliche Faktoren sublimiert werden und die Gruppenleistung reduzieren [Butt82].

Inwieweit sich insgesamt die - zweifellos gegenseitig vorhandenen - Vorurteile und Stereotype äußern, wie mit ihnen umgegangen wurde, ob sie konstruktiv bzw. destruktiv wirkten, war ein wesentlicher Aspekt unserer Untersuchung.

\subsection{Interdependenz und Autonomie}

Grundlegend für Interaktion und damit letztlich für die Gruppenkohäsion ist Interdependenz. Interdependenz ist eine Forderung, die wesentlich über die Aufgabenstellung realisiert werden kann [JoJo91]. Neben den Grundanforderungen an eine problemorientierte, authentische Aufgabenstellung [ReMa99] ist zu beachten, dass die Gruppenaufgabe nicht rein additiv gelöst werden kann. Folgt man der Unterscheidung in kooperatives Lernen, das in stark strukturierten Bahnen verläuft und kollaboratives Lernen, das de facto weitgehend selbstgesteuert funktioniert [ReMa99], so ist zumindest kollaboratives Lernen durch additive Aufgaben kaum zu initiieren bzw. durchzuführen.

Aufgaben für interdisziplinäre Projekte sollten daher so formuliert werden, dass sie nur in Kooperation bewältigt werden können und eine intensive Koordination der Zusammenarbeit notwendig wird. Diese Interdependenz sollte dadurch realisiert werden, dass die Aufgaben intern so aufgeteilt werden, dass Kooperation unumgänglich wird. Ein befriedigendes Ergebnis ist nur zu erzielen, wenn sich alle Gruppenmitglieder an der Lösung der Aufgabe beteiligten.

Kollaboratives Lernen beinhaltet auch, dass die Studierenden z.B. die Möglichkeit besitzen, Aushandlungsprozesse etwa zur Aufgabenverteilung und -strukturierung gruppenintern durchzuführen. Dafür muss die Aufgabe relativ offen gestaltet sein und die Studierenden angemessene Autonomie besitzen. Allerdings gilt sowohl für Autonomie als auch für Interdependenz, dass das Maximum nicht mit dem Optimum zusammenfällt. Werden bei interdependenten Aufgaben die Einzelaufgaben nicht eindeutig verteilt, kann dies zu Redundanz und zu Unzufriedenheit in der Gruppe führen. Grundlegend von Bedeutung ist daher die Möglichkeit zu intensiver Kommunikation und die Absprache und Einhaltung der idealerweise gruppenintern oder aber durch die Betreuer festgelegten Strukturen. Das Prinzip der Angemessenheit gilt in gleichem Maße für die Autonomie der Studierenden bei der Aufgabenbearbeitung. Autonomie führt oft zu Überforderung und Demotivation. Hilfe bei der Strukturierung ist damit unerlässlich. Sinnvoll erscheint es aber, sie adäquat und quasi „on demand” zu leisten, um den Handlungsspielraum nicht von vornherein und pauschal einzuschränken.

Von Interesse für die Untersuchung war, ob und wie die Studierenden mit der Autonomie umgehen konnten, inwieweit es Überforderung gab und wie sich die Interdependenz der Aufgabenstellung auswirkte. 


\section{Konzept}

Das Kooperationsprojekt fand im Wintersemester 00/01 zwischen der Fachhochschule Brandenburg und der Fachhochschule Stralsund statt. Den Gesamtkontext bildete das Bundesleitprojekt „Virtuelle Fachhochschule”, an dem die beiden Hochschulen mitwirken.

In Brandenburg war der Studiengang Wirtschaftsinformatik mit der 4-stündigen Lehrveranstaltung „Informationsmanagement” im Hauptstudium beteiligt. In Stralsund handelte es sich um ein 2 Semesterwochenstunden umfassendes Ergänzungsfach „Virtuelle Teamarbeit”, das für Studierende der Betriebswirtschaftslehre im 4.-6.Semester angeboten wird. Am Modellversuch beteiligten sich 13 Studierende aus Brandenburg und 9 Studierende aus Stralsund.

Zunächst wurden an den Standorten im Präsenzunterricht Grundlagen zu den Gegenstandsbereichen der beiden Lehrveranstaltungen vermittelt. Darüber hinaus wurden die Studierenden in die technische Benutzung der angebotenen Kommunikations- und Kooperationsmittel eingeführt. Die studentische Zusammenarbeit startete Mitte November 2000. Im Verlauf der Zusammenarbeit bildeten sich 5 Arbeitsgruppen mit einer Gruppengröße von je 4-5 Personen.

Zur Unterstützung der studentischen Kooperation wurde in technischer Hinsicht Electronic Mail, das Kommunikationsprogramm ICQ, der Dokumentenserver BSCW, das Videokonferenzsystem Netmeeting und das kooperative System TeamWave angeboten. Hinzu kam das Programm Mindmanager zur Unter-stützung der Ideensammlung in den studentischen Arbeitsgruppen. Zur Archivierung und für den Austausch der gemeinsamen Dokumente wurde BSCW angeboten. Das Videokonferenzsystem Netmeeting 3.01 wurde im Rahmen betreuter Veranstaltungen eingesetzt, die von den Dozenten zentral organisiert wurden. Zur Betreuung der Studierenden kamen ebenfalls elektronische Kommunikationsmedien zum Einsatz. In diesem Anwendungszusammenhang wurde Electronic Mail und ICQ benutzt. Diese Technologien wurden darüber hinaus auch verstärkt zur internen Koordination zwischen den beiden Dozenten eingesetzt.

Für die Unterstützung der Kooperation galt weitgehend das Prinzip der Autonomie. Grundannahme war, dass bei komplexen, interdependenten Aufgaben eine Vielfalt an Kommunikationskanälen vorhanden sein muß. Dementsprechend wurden verschiedene Tools zur Verfügung gestellt, über deren spezifische Nutzung die Studierenden selbst entscheiden konnten.

Als Ausgangspunkt für die Definition der Aufgabenstellung wurde eine Fallstudie aufbereitet und den Studierenden im BSCW bereitgestellt. Um die Authentizität des Szenarios zur gewährleisten, wurde die Aufgabe als Fallstudie angelegt. Die Komplexität der Aufgabe erforderte von den Studierenden multiple Positionen zu untersuchen und zu vertreten. 
Inhalt der Fallstudie war die Frage nach verschiedenen Einsatzmöglichkeiten von CSCW bzw. CSCL in einem global agierenden Unternehmen. Die Studierenden übernahmen die Rolle eines Beratungsunternehmens, das Möglichkeiten zum Einsatz von CSCW-Technologie im Unternehmen zur Unterstützung der Kooperation zwischen verteilten Standorten untersucht. Die Grundlage der gemeinsamen Arbeit war der fiktive Auftrag des Vorstandsvorsitzenden der Beispielfirma. Zusätzliche Informationen wie das Organigramm des Beispielunternehmens sowie Literaturhinweise, aber auch organisatorische Hinweise zum Scheinerwerb, wurden den Studierenden auf dem BSCW Server bereitgestellt. Als inhaltliche Schwerpunkte bei der Bearbeitung der Aufgabenstellung dienten zum einen betriebswirtschaftliche Fragestellungen, zum anderen technische Aspekte der Umsetzung um für beide Seiten Anknüpfungspunkte für die gemeinsame Arbeit zu bieten. Durch die Bearbeitung jeweils unterschiedlicher inhaltlicher Aspekte im weiteren Kontext der Fallstudie bildeten die Hausarbeiten einen inhaltlichen Bezug untereinander.

Unter Beteiligung der Dozenten wurden interdisziplinäre und leistungsheterogene Arbeitsgruppen gebildet. Darüber hinaus sollte eine interessensgeleitete Auswahl der Studierenden hinsichtlich der zu bearbeitenden Aufgabenstellung ermöglicht werden. In diesem Zusammenhang wurden erste Ideen zur Bearbeitung der Aufgabe zwischen den Standorten ausgetauscht und von den Dozenten an den jeweiligen Standorten vermittelt. Anschließend wurden die einzelnen Gruppen zusammengefügt.

Die Gruppenfindung wurde durch eine Videokonferenz in großer Runde unterstuitzt. Vorab wurde den Studierenden an den jeweiligen Standorten angeboten mit dem Videokonferenzsystem zu experimentieren, um Hemmschwellen bei der Techniknutzung abzubauen. Im Whiteboard wurden während der Vorstellungsrunde die Bilder aller Teilnehmer und der Dozenten der virtuellen Lehrveranstaltung zu einem Gruppenbild zusammengefügt (Abb. 4).

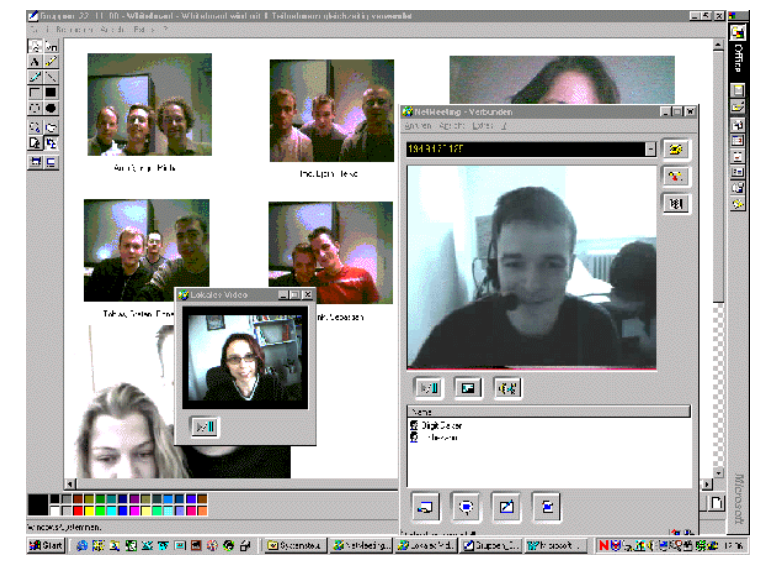

Abbildung 4: Virtuelle Lehrveranstaltung 
In einer zweiten Videokonferenz innerhalb der Arbeitsgruppe fand unter Einsatz des Programms Mindmanager ein Brainstorming zum Zwecke der Aufgabenstrukturierung statt. Diese Videokonferenz fand ohne direkte Beteiligung der Dozenten statt. Aus dieser Sammlung der zu bearbeitenden Aspekte wurde von den Studierenden ein erster Gliederungsentwurf erstellt. Diese Strukturierung wurde durch die Vorgabe der relativ offenen bzw. halb-strukturierten Aufgabenstellung erforderlich. Die Autonomie und die Entfaltungsmöglichkeiten für die Studierenden und die dadurch gewährleistete interessensgeleitete Herangehensweise sollten nicht eingeschränkt werden. Trotzdem schien es notwendig, sowohl zeitlich als auch inhaltlich einen Fixpunkt zu setzen, der den Betreuern die Überprüfung der Vorgehensweise erlaubte.

Zur Beratung und Abstimmung der Gliederung fand eine Sprechstunde via Videokonferenz statt. Auch diese Maßnahme diente der Strukturierung der studentischen Gruppenarbeit und bildete die Grundlage zur Niederschrift der gemeinsamen Hausarbeit.

Die letzte Videokonferenz fand zur Präsentation der Hausarbeit statt. Die Studierenden präsentierten selbständig ihre Hausarbeit und übernahmen in verteilten Rollen auch die Moderation der Veranstaltung.

\section{Ergebnisse}

Die begleitende Evaluation wurde in einer formativen, begleitenden Form durchgeführt. Als qualitative Untersuchungsmethoden wurden Interviews mit den Studierenden und teilnehmende Beobachtung angewendet. Ergänzend wurden die Zugriffe auf das BSCW System ausgewertet. Die folgenden Ergebnisse spiegeln einen Teil der gewonnenen Erkenntnisse wieder.

\subsection{Kooperation und Heterogenität}

Im Rahmen der Fallstudie war grundsätzlich von Interesse, ob es prinzipielle Unterschiede in der Arbeitsweise zwischen den Gruppen gab. Es zeigte sich, dass die größten Probleme bei den Arbeitsgruppen mit sehr unterschiedlichen technischen Vorkenntnissen auftraten. Diese Gruppen beschränkten sich auf wenige Kommunikationstools zum Austausch innerhalb der Gruppe. Primär wurde in diesen Fällen zur Unterstützung der Zusammenarbeit Electronic Mail genutzt. Zudem wurde in den - in Bezug auf die technischen Kenntnisse - sehr divergenten Gruppen das Videokonferenzsystem als synchrones Medium verstärkt genutzt. Die Begründung hierfür ist, dass die Videokonferenzen durch die Dozenten betreut wurden und somit Defizite im technischen Bereich kompensiert werden konnten. Die Archivierung fand in diesen Gruppen meist unabhängig voneinander statt, d.h. die Do- 
kumente wurden via Mail-Attachment an die Gruppenmitglieder mit besseren technischen Kenntnissen geschickt, welche dann die Dokumente in BSCW stellten.

Probleme ergaben sich in einem Fall in Ermangelung eines privaten Internet-zugangs der Studierenden in Stralsund. Die Nutzungsgewohnheiten der Studierenden der Wirtschaftsinformatik, die vorwiegend in den Abendstunden über längere Zeitspannen hinweg online sind, stand dazu in Widerspruch und vereitelte den Einsatz synchroner Medien wie ICQ. Im Rahmen dieser Kooperation wurde im wesentlichen auf das Telefon umgestiegen, da auf ein synchrones Kommunikationsmittel laut Angaben der Studierenden nicht verzichtet werden konnte. Die Studierenden gaben an, daß „Electronic-Mail einfach zu langsam war” und sie insbesondere am Wochenende nicht warten wollten, bis die Gruppenmitglieder in Stralsund erst in der darauffolgenden Woche auf die Mails reagierten.

BSCW war neben der unterstützenden Funktion der Zusammenarbeit in den Arbeitsgruppen auch für die übergreifende Koordination des Projektes von Bedeutung. Intendiert war zudem, dass die Gruppen nicht nur intern, sondern auch miteinander kooperieren. Durch die Anlehnung an die Fallstudie standen die bearbeiteten Aufgabenstellungen inhaltlich zueinander in Beziehung, was eine Zusammenarbeit nahe gelegt hätte. Eine übergreifende Kooperation fand jedoch bis auf wenige informelle Gespräche und wenige Zugriffe auf die Arbeitspapiere und Gliederungen anderer Arbeitsgruppen nicht statt. In diesem Zusammenhang wurden Motive wie ,ich wollt mal sehn, ob wir auf dem richtigen Weg sind” und „war eben interessant, wie die Anderen das machen” angegeben. Bei der Befragung wurde deutlich, dass die Studierenden davon ausgingen, dass eine gruppenübergreifende Kooperation von den betreuenden Dozenten nicht erwünscht war, womöglich sogar als Täuschungsversuch verstanden werden könnte. Es wurde deutlich, dass den Unsicherheiten der Studierenden, die teilweise sehr traditionelle Vorstellungen vom Lehren und Lernen verinnerlicht haben, entgegen gewirkt werden muß.

In den Präferenzen für die verschiedenen Kommunikationsmodi zeigten sich auch deutlich divergente Grundtypen. Eher introvertierte Gruppenmitglieder hatten Schwierigkeiten mit den synchronen Kommunikationsmitteln. Gruppen mit extrovertierten Gruppenmitgliedern kommunizierten dagegen sehr häufig synchron. Weiterhin spielte das jeweilige Gruppenklima eine Rolle bei der Medienauswahl. In den Gruppen, in denen ein vergleichsweise schlechteres Klima herrschte, wurde zu einem größeren Ausmaß asynchron kommuniziert.

\subsection{Explanation, Elaboration und Konflikte}

Von besonderem Interesse hinsichtlich der Forschungshypothesen war, ob sich die positiven Aspekte bezüglich Explanation und Elaboration in den interdisziplinären und leistungsheterogenen Gruppen im gewünschten Maße eingestellt haben. 
Komplementär war ebenso von Interesse, ob sich die Konflikte als stimulierend produktiv oder als leistungsmindernd destruktiv manifestierten.

In diesem Kontext wurden zentrale Unterschiede zwischen den Gruppenteilen deutlich, die wiederum v.a. mit der unterschiedlichen technischen Kompetenz zusammenhingen. In den Gruppen mit großen Unterschieden hinsichtlich der technischen Fertigkeiten wurden teilweise Vorurteile gegenüber der anderen Disziplin geäußert, welche die Studierenden durch ihre Erfahrungen bestätigt fanden. Die WirtschaftsinformatikerInnen verifizierten in erster Linie ihre Stereotype bezüglich der technischen Inkompetenz ihrer Gruppenmitglieder. Auf der anderen Seite fühlten sich die BetriebswirtschaftsstudentInnen in ihren Vorurteilen über die mangelnden sozialen Fähigkeiten der InformatikerInnen bestätigt. Diese Vorurteile führten allerdings nicht zu offenen Konflikten. Das Arbeitsklima wurde dadurch relativ wenig belastet.

Die Explanation und Elaboration fand nicht im gewünschten Ausmaß statt. Die Studierenden waren auf ihren Fachgebieten jeweils zu überlegen und übten diese Dominanz auch aus. Unterstützung durch die technisch kompetenteren Studierenden wurde nur in den synchron unterstützten Kooperationssituationen beobachtet. Die Endkorrektur der Hausarbeiten wiederum wurde weitgehend durch die BWLStudentInnen übernommen, die meist über einen besseren sprachlichen Ausdruck verfügten.

Die Auseinandersetzung mit den Inhalten des anderen Teils der Gruppe und der damit verbundenen Perspektive geschah größtenteils über das Lesen der Texte und nur teilweise über Diskussionen in den Videokonferenzen. Inhaltliche, aufgabenbezogene Diskussionen im Sinne von Explanationsprozessen waren sehr unterschiedlich verteilt. Hier spielten Motivation und Interesse an der Aufgabe, vor allem aber an den anderen Teilnehmern eine zentrale Rolle. War dieses Interesse gering, reduzierte sich die Explanation auf die zur Aufgabenlösung notwendigen Aspekte. Insgesamt wuchs aber das Interesse und die Motivation in allen Gruppen kontinuierlich, so dass sich die Kooperation mit zunehmender Zeit besser gestaltete und Explanationsansätze häufiger wurden.

\subsection{Interdependenz und Autonomie}

Der gruppeninterne Austausch zur Aufgabe und ihrer Strukturierung war zumindest zu Beginn nicht unproblematisch. Teilweise waren die Studierenden in Stralsund mit den technischen Fachtermini im speziellen und allgemein mit der in Brandenburg bereits in Ansätzen ausgearbeiteten Aufgabenstellung überfordert. Teilweise konzentrierten sie sich dann anfangs auf die ihnen zugewiesenen Gebiete und zeigten wenig Interesse für die Gesamtaufgabe. Andere Gruppenmitglieder erarbeiteten die Aufgabe kooperativ bzw. die Studierenden in Stralsund erkundigten sich explizit nach den Zusammenhängen. Mit zunehmendem Interesse an der Aufgabe und der Gruppe arbeiteten sich auch die Gruppenmitglieder in 
Stralsund, die ihre Aufgabe zunächst von Brandenburg aufoktroyiert bekamen, in die Gesamtaufgabe ein und modifizierten sie.

Die Dominanz der Studierenden in Brandenburg wurde in den Befragungen mit der unterschiedlichen curricularen Einbettung der Lehrveranstaltungen an den beiden Standorten begründet. Da der Kurs für die Studierenden in Brandenburg für ihr Studium von größerer Bedeutung war, waren diese auch bereit im größeren Umfang Koordinationsaufgaben zu übernehmen. Die Stralsunder Studierenden akzeptierten diese Aufgabenteilung.

Interdependenz bis hin zu Redundanz gab es insbesondere bei den allgemeinen Teilen der Aufgabe, etwa bei Begriffsbestimmungen und Definitionen. Hier arbeiteten die Studierenden teilweise parallel. Im zeitlichen Verlauf der Kooperation versuchten die StudentInnen jedoch sukzessive, die gewünschte Perspektivenverschränkung herbeizuführen.

\section{Fazit}

Das aus konstruktivistischen Annahmen abgeleitete Prinzip der Selbststeuerung und Autonomie ist sehr diffizil zu handhaben. Die Balance zwischen Unterforderung durch zu stark strukturierte und Überforderung durch mangelhaft strukturierte Aufgaben, muss auch während der Lehrveranstaltung ständig neu austariert werden. Zudem zeigten sich bei einer weitgehend offenen Aufgabenstellung eine Reihe von Anlaufschwierigkeiten. Da die Kooperation im Laufe der Zeit in allen Gruppen immer intensiver wurde, wäre zu überlegen, den zeitlichen Rahmen auszudehnen.

Die Anlehnung der Aufgabenstellung an eine Fallstudie hat sich im Verlauf der beschriebenen Kooperation bewährt. Dennoch sollten inhaltliche Vorgaben als optionale Möglichkeit für die Ausarbeitung der Fragestellung angeboten werden. Kreative Gruppen waren durchaus in der Lage, sich ihre Aufgabenstellung auf individuelle Weise $\mathrm{zu}$ erschließen und fühlten sich teilweise durch die Vorgaben verpflichtet, einen Bezug zur Fallstudie künstlich herzustellen.

Realisiert wurde die Strukturierung vor allem durch die vorgegebenen Videokonferenzen. Die vier Videokonferenzen schafften einen organisatorischen Rahmen und stärkere Verbindlichkeit für die virtuelle studentische Gruppenarbeit. Insgesamt halten wir den Einsatz von synchronen Kommunikationsmitteln im Verlauf einer räumlich verteilten Kooperation als wesentliche Determinante für den Erfolg eines solchen Vorhabens.

Prinzipiell bleibt das Konzept einer weitgehend offenen Aufgabenstellung sowohl angesichts der Ergebnisse wie auch der überwiegend positiven Einschätzungen durch die Studierenden sinnvoll. 
Ein wesentlicher Punkt bei der Formulierung der Aufgabenstellungen ist neben der Beachtung von Authentizität und Vorwissen der Studierenden die Berücksichtigung des organisatorischen Rahmens. Der unterschiedliche Stundenumfang der Lehrveranstaltungen an den beiden Standorten legte die Dominanz der Studierenden in Brandenburg nahe und verhinderte so gleichzeitig einen intensiveren und gleichberechtigteren Austausch sowie teilweise auch die intendierten Explanationsprozesse. Hinzu kam der Vorsprung der WirtschaftsinformatikerInnen in Hinsicht auf die technischen Fertigkeiten.

Insgesamt mussten die Erwartungen an intensive, durch die Interdisziplinarität implizierte gruppeninterne Prozesse teilweise revidiert werden. Allerdings gab es auch keine durch die latent vorhandenen Stereotype hervorgerufenen Konflikte.

Ebenso sinnvoll wie die Autonomie bei der Aufgabenstrukturierung war das Konzept der Bereitstellung unterschiedlicher Kommunikationsmittel. Die gruppenspezifische Nutzung der verschiedenen Tools ermöglichte Kooperations- und Kommunikationsprozesse auf diversen, den Bedürfnissen der Studierenden angepassten Wegen. Insbesondere der hohe Anteil an synchroner Kommunikation half den jeweils sehr unterschiedlichen Gruppenmitgliedern zunehmend mehr Kohäsion in der Gruppe zu erzeugen.

Für die Betreuer war die beschriebene Kooperation mit hohen Anforderungen an die interne Koordination verbunden. Allein der Aufwand für die Absprachen zu terminlichen und inhaltlichen Aspekten war weitaus größer als angenommen.

Die abschließende Frage, ob es mehr Reibungsverluste gegeben hat oder die Gewinne durch die Synergie überwogen, lässt sich nicht eindeutig beantworten. Die Studie zeigte, dass die positiven Effekte durch die Gestaltung der Faktoren wie Heterogenität und Autonomie teilweise nur schwer zu erzielen sind bzw. von anderen, beispielsweise organisatorischen Aspekten überlagert werden.

Zieht man für den Lernerfolg als Vergleich die Arbeiten, die in Präsenzgruppen in Brandenburg in der Lehrveranstaltung Informationsmanagement entstanden sind, heran, so ergaben sich keine Qualitätsunterschiede. Vielmehr waren die im CSCLProjekt erstellten Arbeiten vielfältiger und perspektivenreicher.

Der Erfolg von CSCL umfasst nicht allein den in den Hausarbeiten deutlich werdenden Lernerfolg, sondern auch Aspekte wie die gewissermaßen en passant erlernten tele-kommunikativen Fähigkeiten. Gerade das Erlernen des Umgangs mit den neuen Kommunikationsmedien wurde in der Evaluation von den Studierenden auch explizit angesprochen und als positiv bewertet. $\mathrm{Zu}$ den positiven Aspekten muss ebenfalls die von den Studierenden geäußerte subjektive Zufriedenheit miteinbezogen werden. Für die Lehrveranstaltung kann daher insgesamt ein positives Fazit gezogen werden. 


\section{Literatur}

[Aue98] Auer-Rizzi, W.: Groupthink. In: Die Betriebswirtschaft, (1998), 5, S. 677-680.

[Butt82] Butterworth, G.: A brief account of the conflict between the individual \& the social in models of cognitive growth. In: G. Butterworth \& P. Light (Eds) Social Cognition. Brighton, Sussex 1982, pp. 3-16.

[Dill95] Dillenbourg, P.; u.a.: The evolution of research on collaborative learning. P. Reimann \& H. Spada (Eds.) Learning in humans and machines. Towards an interdisciplinary learning science. Oxford 1995, pp. 189-211.

[DiSc95] Dillenbourg, P. \& Schneider, D.: Collaborative learning and the Internet. http://tecfa.unige.ch/tecfa/research/CMC/colla/iccai95_1.html (1995). [17.08.1999].

[JoJo91] Johnson, D.W. \& Johnson, R.T.: Learning together and alone: Cooperative, competitive, and individualistic learning. Englewood Cliffs 1991.

[MaRe92] Mandl, H. \& Renkl, A.: A plea for „more local” theories of cooperative learning. In: Learning and Instruction 2, (1992) S. 281-285.

[Piag26] Piaget, J.: Das Erwachen der Intelligenz beim Kinde. Stuttgart 1991.

[ReMa99] Reinmann-Rothmeier, G. \& Mandl, H.: Teamlüge oder Individualisierungsfalle? Eine Analyse kollaborativen Lernens und deren Bedeutung für die Förderung von Lernprozessen in virtuellen Gruppen. Ludwigs-Maximilians-Universtität, Institut für Pädagogische Psychologie, Forschungsbericht Nr. 115, München 1999.

[Salo95] Salomon, G.: What Does the Design of Effective CSCL Require and How Do We Study Its Effects? URL: http://www.cica.indiana.edu/csc195/outlook/62_Salomon.html (1995), [12.09.2000].

[Slav93] Slavin, R. E.: Kooperatives Lernen und Leistung: Eine empirisch fundierte Theorie In: G. Huber (Hg.): Neue Perspektiven der Kooperation. Ausgewählte Beiträge der Internationalen Konferenz über kooperatives Lernen (Grundlagen der Schulpädagogik; 6), Baltmannsweiler 1993, S. 151-170.

[Vygo62] Vygotsky, L.S.: Thought and Language. Cambridge, Massachusetts, 1962.

[Webb91] Webb, N.M.: Task related verbal interaction and mathematics learning in small groups. Journal for Research in Mathematics Education, 22 (5), 1991, pp.366-389. 